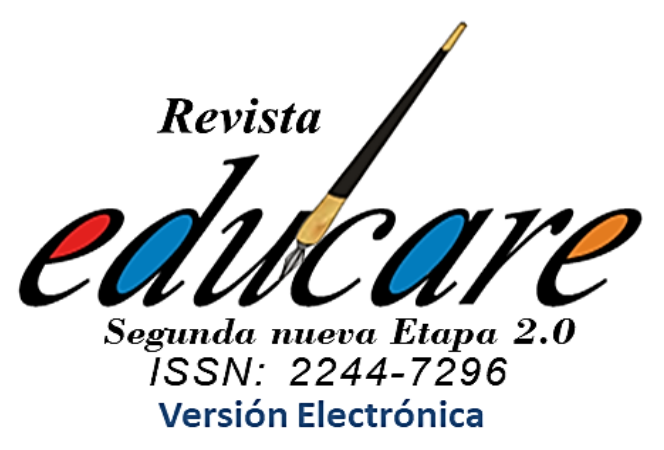

Volumen 24 № 3Septiembre-Diciembre 2020

$(111-137)$

María Alejandra Martínez Barrientos*

ORCID:http://orcid.org/0000-0001-5903-7295

Joaquín Gairín Sallán*

ORCID:https://orcid.org/0000-0002-2552-0921

UNIVERSIDAD CATÓLICA BOLIVIANA

UNIVERSIDAD ADVENTISTA DE BOLIVIA

*Psicóloga con Maestría en Educación Superior (U Sherbrooke, Canadá), Diploma de Estudios Avanzados en Psicología Social (U. Granada, España) y Doctoranda en Educación de la UAB. Actualmente es directora del Departamento de Educación de la Universidad Católica Boliviana. Correo Electrónico: mmartinez@ucb.edu.bo

**Profesor de EGB, Graduado social, Psicólogo y Pedagogo, actualmente es decano de facultad, comisionado del Clúster en Educación y

Formación, director de departamento universitario y del ICE de la UAB. Catedrático de Didáctica y Didáctica y Organización escolar. Correo electrónic: joaquin.gairin@uab.es
REPÚBLICA BOLIVARIANA DE VENEZUELA UNTVERSIDAD PEDAGÓGICA EXPERIMENTAL LIBERTADOR INSTITUTO PEDAGÓGICO DE BAROUISIMETO "LUIS BELTRÁN PRIETO FIGUEROA" SUBDIRECCIÓN DE INVESTIGACIÓN Y POSGRADO BARQUISIMETOESTADO LARA

\section{ACTITUDES HACIA LA VALIDACIÓN DE APRENDIZAJES PREVIOS Y SU INCLUSIÓN EN EL SISTEMA UNIVERSITARIO BOLIVIANO}

\section{VALIDATION OF PREVIOUS LEARNING AND ITS INCLUSION INTO THE UNIVERSITY BOLIVIAN SYSTEM}

Recibido:

20-07-2020

Aceptado:

10-10-2020 


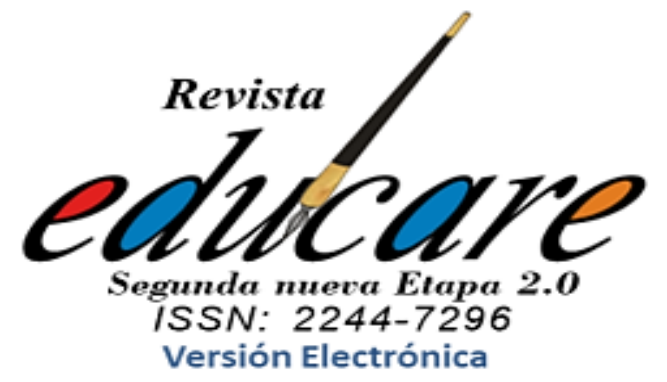
"LUIS BELTRÁN PRIETO FIGUEROA

\section{Resumen}

Este estudio analiza las actitudes de instituciones universitarias de La Paz, Bolivia hacia procesos de validación de aprendizajes previos (VAP) y las experiencias que ya se han desarrollado. La teoría de Fishbein y Azjen sobre actitudes, permite analizar los factores para incluir poblaciones no tradicionales en itinerarios profesionalizantes. Se aplicó una encuesta para identificar carreras candidatas a VAP y un cuestionario para identificar actitudes de los actores vinculados a estos procesos. Los resultados muestran equilibrio las actitudes en favor y en contra, presentando las diferencias más relevantes en carreras técnicas y algunas en ciencias sociales y humanas como Comunicación y Psicopedagogía. Se concluye que aceptar estos procesos podría facilitar a las universidades abrir espacios para democratizar la educación superior reconociendo la experiencia empírica del aprendizaje no formal e informal, impactando el desarrollo social al promover el aprendizaje permanente y la profesionalización del recurso humano a niveles cada vez más elevados.

Palabras clave: Educación superior; aprendizaje a lo largo de la vida; actitudes; aprendizajes previos.

\section{Abstract}

This study analyzes university institutions' attitudes in La Paz, Bolivia towards VAP processes, and the experiences that have already been developed. Fishbein and Azjen's theory on attitudes allows us to analyze the factors for including non-traditional populations in professionalizing itineraries. A survey was applied to identify candidate careers for VAP and a questionnaire to identify the actors' attitudes linked to these processes. The results show a balance of attitudes for and against, presenting the most relevant differences in technical areas of study and social and human sciences such as Communication and Psychopedagogy. It is concluded that accepting these processes could facilitate universities to open spaces to democratize higher education, recognizing the practical experience of non-formal and informal learning, impacting social development by promoting lifelong learning and the professionalization of human resources at increasingly higher levels.

Keywords: Higher education; lifelong learning; high education; inclusion, attitudes; accreditation of prior learning. 


\section{INTRODUCCIÓN}

El mundo ha ingresado en un proceso histórico de cambios rápidos y profundos en todas las actividades de los seres humanos, incluyendo la educación superior y sus instituciones. Estos cambios requieren una transformación de las posturas personales y, por lo tanto, están supeditados, entre otros factores, a las actitudes de estos actores (Casanova, Canquiz, Paredes e Inciarte, 2018).

Entre estos cambios se encuentra el planteamiento que indica que la educación debería acompañar a la persona durante toda su vida, Lifelong learning (Dávila, 2013). Esto se explicita en Nairobi (1976), donde se plantea la necesidad de pensar en el aprendizaje a lo largo de toda la vida en todos los niveles educativos, incluyendo a la educación superior. Supone, además, considerar que el ser humano aprende más allá de los espacios formales y obliga a hablar también de los aprendizajes no formales (aquél que se logra de manera consciente en un espacio con una relativa estructura curricular) e informales (desarrollado en el espacio del trabajo, el ocio o la recreación, muchas veces de manera inconsciente) (Fernández, 2014; Casanova et al., 2018).

Las instituciones distan mucho de considerar aquellos aprendizajes no formales e informales; sin embargo, la Organización de las Naciones Unidas para la Educación, la Ciencia y la Cultura (Unesco) y la Organización para la Cooperación y el Desarrollo Económico (OCDE) han planteado la necesidad de modificar esta situación y así lo han propuesto en varias oportunidades y documentos. En esta línea, la Unión Europea ha dado pasos significativos para revertir y modificar una estructura educativa que sólo visibiliza lo formal, ello a través de la creación del Centro Europeo para el Desarrollo de la Formación Profesional (CEDEFOP) que responde a esta demanda; así como el Marco Europeo de Calificaciones para el Aprendizaje Permanente (MEC), que señala 8 niveles de conocimientos, destrezas y competencias que van desde un nivel muy básico hasta un nivel de doctorado, los niveles 5-8 se corresponden con los descriptores de educación superior acordados en el Proceso de Bolonia (CEDEFOP, 2009).

Estos niveles viabilizan el planteamiento de procesos de Validación de Aprendizajes Previos (VAP, en adelante).

Sin embargo, en Latinoamérica en general, y en Bolivia en particular, la relación de estos aprendizajes con lo que es valorado o reconocido por los recursos humanos de una institución está poco definido. Esta falta de inclusión deja a miles de personas fuera de la 
educación formal y de la posibilidad de mayor desarrollo profesional y, por lo tanto, de poder aportar al crecimiento social y económico de la región. Esta es la motivación que orienta la presente investigación, indagar sobre las posibilidades de abrir nuevos espacios y oportunidades para poblaciones tradicionalmente excluidas de la educación superior, considerar a nuevos beneficiarios y a nuevas formas de plantear el proceso de enseñanza-aprendizaje.

Esta apertura requiere de un cambio de actitudes, tanto en los potenciales favorecidos por estos procesos como en los decisores y ejecutores de nuevas alternativas educativas, especialmente de los procesos de VAP, para llegar a generar la inclusión y contribuir a la democratización de la educación superior

\section{ARGUMENTACIÓN TEÓRICA}

La aceptación de que la educación superior debe cimentarse en el enfoque de formación basada en competencias es un logro bastante consolidado, que responde a la necesidad de adecuar de manera pertinente los procesos de formación profesional a las demandas del contexto y a una pedagogía más acorde al desarrollo del conocimiento en el siglo XXI (Tobón, 2008; Casanova y cols., 2018). Así lo plantea también la Unesco en la Segunda Conferencia Mundial sobre Educación Superior realizada en París (2009), donde subraya la necesidad de formar competencias sólidas para enfrentar el mundo de hoy y el del mañana.

Los procesos de VAP han sido desarrollados desde hace varias décadas por diferentes países, siendo la Unión Europea la que ha llevado la iniciativa con experiencias muy puntuales hacia mediados del siglo pasado, hasta lograr consolidar un sistema con directrices que han permitido que, aproximadamente 150 países de los 193 adscritos a Naciones Unidas, hayan desarrollado marcos de cualificación (3 de cada 4) inspirados en los del European Qualification Framework (EQF,en adelante) e instrumentos que permiten viabilizar los procesos de VAP.

México es uno de los países en Latinoamérica que ha marcado tendencia con el Consejo Nacional de Normalización y Certificación de Competencias Laborales (CONOCER), con más de 20 años de experiencias exitosas que han logrado vincular la certificación de competencias laborales con las instancias de formación que llegan hasta el nivel de educación terciaria a nivel de grado, favoreciendo las salidas intermedias y abriendo posibilidades de empleabilidad que favorecen el desarrollo productivo. En otros países de la región, se identifica el Servicio Nacional de Aprendizaje (SENA) en Colombia, ChileValora en Chile, el Programa Nacional 
para la Promoción de Oportunidades Laborales (Impulsa) de PERÚ), todos ellos apuntando a que la certificación de competencias y la formación/capacitación logren llegar a cada vez más altos estándares y particularmente en instituciones de formación técnica.

Asimismo, se cuenta con informes nacionales, internacionales, encuentros, reuniones y otros eventos que permanentemente exponen los logros de los diferentes países en esta temática. Tal es el caso de las Bienales organizadas por Centerof Lifelong Learning Services (CL3S) que ya han realizado 3 versiones, la última en mayo de 2019, y que han ido incluyendo las experiencias de países de distintas partes del mundo: desde los países nórdicos, pasando por Sud África y llegando hasta Venezuela (Duvekot, Coughlan y Aagaard, 2017). Otro espacio que ha sido creado explícitamente para dar cabida a las experiencias de VAP es el CEDEFOP cuyo informe de 2019, también en su tercera versión, incluye las experiencias de 84 países de los cinco continentes, siendo destacable que sólo esté presente México de Latinoamérica.

La experiencia de este país ha logrado integrar tanto la certificación de competencias y su acreditación con procesos formativos a diferentes niveles, particularmente en educación técnica a nivel medio y superior. Y se encuentra en la búsqueda de nuevos itinerarios formativos para lograr el reconocimiento por parte de las universidades de un Marco Nacional de Cualificación con 8 niveles similar al europeo y un Banco de Créditos Académicos (Unesco, 2019).

Las prácticas que se han desarrollado en varios países para dar cabida a procesos de VAP, son innumerables y se desarrollan en diferentes niveles de formación. Entre algunas de estas experiencias se observa el caso de maestrías en Dinamarca, que impulsan la posibilidad de obtención de competencias reconocidas formalmente basándose en aquellas adquiridas tanto en el ámbito vocacional específico como las competencias de educación formal. Estas competencias deben tener el mismo nivel de desarrollo que las logradas a través de procesos tradicionales (Wahlgren enSingh, 2015).

En Inglaterra, la mayor cantidad de investigaciones sobre la VAP se han desarrollado sobre los procesos requeridos para la acreditación de créditos para educación superior. Así, los estudios muestran un porcentaje importante de universidades que han introducido procedimientos para desarrollar VAP; sin embargo, el impacto real aún no ha sido significativo en términos de población que accede a las universidades a través de estos procesos (Pokorny en Harris, Breier, and Wihak, 2011). Los mayores éxitos, particularmente los referidos a los 
procesos de evaluación de las competencias logradas a través de aprendizajes informales o no formales, están en áreas como las ingenierías, las ciencias naturales, tecnología y diseño, donde es más fácil encontrar las evidencias sobre lo realizado en un nivel empírico.

Asimismo, investigaciones realizadas en Estados Unidos muestran que, pese a las barreras que dificultan la aceptación de procesos de VAP en instituciones universitarias con conceptos tradicionales sobre educación, aprendizaje y el rol que juegan estudiantes adultos en la misma y las actitudes en general, las experiencias logradas denotan que los estudiantes que han participado en la evaluación de sus aprendizajes previos tienen un rendimiento superior comparado con estudiantes tradicionales (Travers en Harris et al., 2011).

Aunque los procesos de VAP existen hace varias décadas en muchos contextos, se trata de una temática relativamente nueva en países como Bolivia. Pese a la existencia del Sistema Plurinacional de Certificación de Competencias (SPCC), que certifica justamente esos aprendizajes no formales o informales, la certificación de competencias no ha incursionado aún en la vinculación entre certificación y formación (Ministerio de Educación, 2017).

En la ciudad de La Paz, principal ciudad de Bolivia, existen tres universidades pertenecientes al Sistema de Universidades Boliviano (SUB): Universidad Mayor de San Andrés (UMSA), Escuela Militar de Ingeniería (EMI) y Universidad Católica Boliviana San Pablo (UCB). Ninguna ha desarrollado experiencias concretas en VAP, aunque se registran algunos intentos como el reconocimiento de bibliotecarios empíricos para que continúen su formación a nivel de licenciatura, o la acreditación de campesinos en el área de agronomía para continuar en el técnico superior, de personas dedicadas a los cuidados de la salud y su reconocimiento para el título de enfermeras en la UMSA, o la posibilidad de aceptar a personas con experiencia en educación parvularia para continuar la carrera de psicopedagogía en la UCB. Estas experiencias resultaron fallidas y no concluyeron el proceso; entre las razones que se identifican son las actitudes de los actores implicados en la toma de decisiones, particularmente los académicos que han frenado estas iniciativas e impedido que estos procesos de VAP se lleven adelante exitosamente, actitudes que requieren la apertura hacia incluir en la universidad a poblaciones no tradicionales (Quevedo, 2019; Mercado, 2019).

El concepto de actitud ha sido, al respecto, principalmente desarrollado por la Psicología Social desde Allport (1930 en Moscovici, 1991) hasta Jain (2014 en Demichelli y García, 2016), dejando claro que este constructo influye en todos los ámbitos de acción del ser 
humano. En este sentido, se hace preciso estudiar y caracterizar la comprensión de estas actitudes para generar propuestas de intervención educativa que favorezcan avanzar hacia una sociedad más democrática.

Para hablar de actitudes, se ha considerado la Teoría del Comportamiento Planificado Theory of Planned Behaviour (TPB) de Ajzen y Fishbein (1975). Estos autores propusieron un planteamiento alternativo al modelo tridimensional (Ajzen y Fishbein, 2000; Ajzen, 2011; Sulbarán, 2009; Steinmetz, Knappstein, Ajzen, Schmidt y Kabst, 2016). De acuerdo con la TPB, las intenciones y, por lo tanto, las conductas, están en función de tres determinantes básicos, uno de naturaleza personal, la intención comportamental; otro que refleja la influencia social, las normas subjetivas; y un tercero que está vinculado a los factores de control, las creencias de control. Así, para algunas actuaciones e intenciones, unos componentes pueden tener mayor influencia que otros, dependiendo de las circunstancias.

La relación se da entre la conducta que está definida por la intención de conducta y, ésta a su vez, por la actitud y la norma subjetiva. La norma subjetiva, a diferencia de la actitud, trata de la evaluación sobre un objeto, pero desde la perspectiva grupal. Incluye, también, todas las representaciones cognitivas acordadas socialmente sobre los pensamientos, sentimientos y actuaciones posibles frente a una situación u objeto actitudinal (Ubillos, Mayordomo y Páez, 2003).

La fuerza subjetiva que tenga una creencia se correlaciona con la posibilidad de que esa actitud derive en una conducta de igual valor a la actitud. Cada creencia está asociada a un atributo y la actitud hacia el objeto está determinada por los valores subjetivos o evaluaciones de los atributos asociados con el objeto y por la fuerza de estas asociaciones. Desde este modelo, la construcción de las actitudes se da de manera espontánea y se modifica de esta misma manera en tanto el sujeto adquiere más información respecto al objeto y en cuanto los valores subjetivos respecto a los atributos del objeto actitudinal se vinculan con el objeto en sí mismo (Ajzen y Fishbein, 2000).

La TPB (Ajzen y Albarracín, 2007) incluye la percepción del control del sujeto sobre su comportamiento y sobre el contexto, intentando predecir la relación entre actitudes y conducta aún en aquellas situaciones que están fuera del control del sujeto, entendiendo esta percepción como un elemento central de esta posibilidad de predicción. 
Esta teoría ha sido utilizada en diversos estudios y con un amplio espectro de campos donde se ha aplicado. Los más frecuentes han sido estudios realizados en comportamientos de ciudadanía responsable, predicción de conductas de alimentación sana, realización de ejercicios, hábitos de cuidado de presión arterial, consumo de drogas, consumo de servicios, comportamiento de cuidado al medio ambiente y comportamientos de autocuidado (Hagger, Chan, King Chung, Protogerou y Chatzisarantis, 2016; Fichten, Amsel, Jorgensen, Nguyen, Budd, Havel, King, Jorgensen y Asuncion, 2016; Liobikiene, Mandravickaite, y Bernatoniene, 2016) entre algunos de los muchos dominios donde esta teoría ha sido utilizada. Es relevante mencionar que muchos de estos estudios no se han centrado únicamente en la evaluación de la actitud en términos del constructo que precede al comportamiento, sino que, además, han demostrado efectividad en la intervención hacia el cambio, particularmente aquellos que se han desarrollado desde una perspectiva más grupal que individual (Steinmetz, et al., 2016). De esta manera, la TPB resulta un marco teórico sólido para comprender, predecir y, más adelante, poder intervenir hacia el cambio y la aceptación de procesos de VAP.

En función de todo lo anterior, la presente investigación plantea, el siguiente objetivo general: Analizar las actitudes de los actores universitarios sobre la inclusión de procesos de validación de aprendizajes previos en el Sistema Universitario Boliviano. Como objetivos específicos se plantean: a) identificar posibles carreras para la implementación de procesos de VAP; b) analizar la percepción que tienen los actores de las carreras identificadas respecto a la VAP en Bolivia y el mundo; y c) identificar las características de las actitudes de los actores respecto a procesos e incorporación del VAP en el Sistema universitario boliviano.

\section{ASPECTOS METODOLÓGICOS}

La metodología de este estudio se enmarca en el método descriptivo cuantitativo, se trata de describir la realidad a partir de los datos cuantitativos medibles por medios válidos y confiables y a partir de la deducción lógica; utiliza un método de análisis sistemático y cuantificable en términos de los instrumentos y técnicas elegidos para el relevamiento de la información (Bharadwaj, 1996; López, 2006 y Bizquera, 2009).

Se utiliza un procedimiento secuencial que se inicia con la identificación de contextos y agentes donde focalizar el estudio. La población de estudio la constituyen los actores 
pertenecientes al SUB en la ciudad de La Paz identificando tres unidades de estudio inicial: UMSA, EMI y UCB.

El diseño metodológico de la investigación se organizó en cuatro etapas, en cada una de las cuales se especifican los instrumentos, fuentes de información y tipo de muestreo desarrollado. Cada caso se describe a continuación:

- Etapa A: Encuesta para identificar carreras potencialmente candidatas para VAP y recoger la opinión sobre la viabilidad de su implementación para el acceso a las carreras. Se aplica a Directores/ Jefes Estudio de carreras de tres universidades. En este caso se consideraron informantes clave con un muestreo de casos políticamente relevantes. Se tienen, por lo tanto, 50 carreras en la UMSA, 14 carreras en la EMI y 20 carreras en la UCB. Se logró alcanzar el 100\% de carreras de la UMSA, EL $100 \%$ de carreras de la UCB y a algunas de la EMI.

- Etapa B: Construcción y validación de un cuestionario con base en escalas de estimación directa. Se aplica a tres expertos nacionales y tres internacionales bajo la lógica de muestreo por casos extremos.

- Etapa C: Cuestionario de estimación directa para conocer e identificar las actitudes y sus componentes de los actores vinculados a potenciales procesos de VAP. Se aplica a Docentes universitarios (UMSA, EMI, UCB). seleccionados a partir de la identificación en la etapa A, en aquellas carreras potencialmente interesadas en procesos de VAP de aprendizajes previos.

Se trata de un muestreo por criterio lógico (Quintana, 2006). En este tipo de muestreo, la investigación define ciertos criterios identificados a través de las encuestas aplicadas y se trabaja únicamente con estos.

Para esta etapa, la EMI no concluyó su participación, por lo que se contó en total con 54 académicos de la UCB que es igual a 56,8\% del total de docentes de las carreras seleccionadas y a 41 docentes de la UMSA, lo que equivale a 43,2\% de docentes de las carreras seleccionadas.

- Etapa D: Procesamiento de la información

Las variables de estudio se identifican y describen a continuación, así como sus dimensiones e indicadores (Artigas y Robles, 2010 y Arias, 2012): 
A. Validación de Aprendizajes Previos: Proceso que reconoce, valida y acredita competencias empíricas, para incorporar a un estudiante en condición de alumno regular en una licenciatura, pese a no tener antecedentes académicos formales. Consideran las siguientes dimensiones:

A.1. Enfoque del Currículum: identificación de aquellas carreras cuyo proyecto curricular se ha formulado desde el enfoque de formación basado en competencias. Se aplica la encuesta.

\section{A.2. Apertura a procesos de VAP}

\section{A.3. Viabilidad Normativa}

\section{A.4. Viabilidad Económica}

\section{A.5. Viabilidad Técnica}

B. Actitudes hacia procesos de VAP en el SUB: Predisposición aprendida para responder a un objeto de forma positiva o negativa, tomando en cuenta la percepción que tiene el sujeto respecto a la ejecución o rechazo de comportamientos vinculados a procesos de VAP. Considera las dimensiones de:

B.1. Intención comportamental: Se evalúan las creencias sobre procesos de VAP, en tanto la apertura, la concepción de la educación como un derecho, viabilidad de VAP y los recursos requeridos para su implementación.

B.2.Norma subjetiva: En este caso, las personas relevantes son las autoridades, los docentes, los estudiantes, la institución en sí, los profesionales y la sociedad en su conjunto. Se aplica el cuestionario.

B.3. Creencia de control: Se considerarán aspectos como la apertura hacia la implementación de VAP, la viabilidad de la evaluación, la normativa respectiva y lo recursos técnicos necesarios. Se aplica el cuestionario.

Los instrumentos utilizados, según la descripción anterior son: a) Encuesta para identificar carreras potencialmente candidatas para VAP y b) Cuestionario de estimación directa para evaluar las actitudes hacia procesos de VAP

La encuesta se caracteriza por preguntas cerradas dicotómicas, sobre cuestiones concretas, con función de filtro, precodificadas y autoadministradas. Su objetivo es el de identificar y facilitar la toma de decisiones respecto a las carreras potencialmente viables e interesadas en aplicar procesos de VAP. Este instrumento responde a las especificaciones 
definidas en la variable A. Validación de Aprendizajes Previos. El criterio principal verificado en la encuesta para elegir las carreras que potencialmente podrían estar interesadas en procesos de VAP, fue que el proyecto académico esté diseñado desde el enfoque de formación por competencias.

Basándose en las respuestas a este criterio, se organizaron el resto de los reactivos en dos grupos, haciendo un total de tres grupos de preguntas:

- Grupo P1, P4: relativo al currículo académico (filtro inicial).

- Grupo P2, P3: relativo a la consideración formal y la apertura para aplicar VAP.

- Grupo P5, P6 y P7: relativa a la viabilidad.

Una vez construidos estos grupos se obtiene una variable con la combinación lineal, de acuerdo con la siguiente formulación:

$$
\sum_{i=1}^{3} \sum_{j=1}^{n_{i}} w_{i j} x_{i j}
$$

Donde:

$\mathrm{w}_{\mathrm{i}}$ : Es el peso de cada uno de los grupos de preguntas $\mathrm{i}=1,2,3$

$\mathrm{x}_{\mathrm{ij}}$ : El valor de la respuesta de cada i-ésima pregunta con $\mathrm{j}=1,2, \mathrm{n}$

En la tabla 1 se presentan los valores de las respuestas y los pesos para cada una:

\section{Tabla 1}

Valores asignados a las respuestas de la encuesta para la toma de decisiones sobre las carreras que pasarán a la etapa siguiente

\begin{tabular}{|c|c|c|c|c|}
\hline GRUPOS & VALOR DE LAS RESPUESTAS & VALOR & PESO* & VALOR x PESO \\
\hline \multirow{4}{*}{$\mathrm{P} 1+\mathrm{P} 4$} & Ambas respuestas negativas & 0 & 0 & 0 \\
\hline & $\begin{array}{l}\text { P1 Afirmativas hacia objetivos y } \\
\text { negativa para P4 }\end{array}$ & 1 & 10 & 10 \\
\hline & $\begin{array}{c}\text { P1 Afirmativas hacia competencias y } \\
\text { negativa } \mathrm{P} 4\end{array}$ & 2 & 10 & 20 \\
\hline & $\begin{array}{l}\text { P1 Afirmativas hacia competencias y } \\
\text { positiva P4 }\end{array}$ & 3 & 23,3 & 70 \\
\hline \multirow{3}{*}{$\mathrm{P} 2+\mathrm{P} 3$} & Ambas negativas & 0 & 0 & 0 \\
\hline & Solamente una afirmativa & 1 & 40 & 40 \\
\hline & Ambas positivas & 2 & 60 & 120 \\
\hline \multirow{4}{*}{$\mathrm{P} 5+\mathrm{P} 6+\mathrm{P} 7$} & Ninguna afirmativa & 0 & 0 & 0 \\
\hline & Solamente una positiva & 1 & 20 & 20 \\
\hline & Solamente dos positivas & 2 & 30 & 60 \\
\hline & Las tres positivas & 3 & 40 & 120 \\
\hline
\end{tabular}

Nota: *Los pesos son subjetivos instalados según los criterios favorables a la incorporación del VAP. Elaboración propia. 
Una vez obtenida la variable, se decidió establecer la mediana (250) como punto de corte. Quedaron 52 carreras a las que se aplicó el siguiente instrumento.

El cuestionario de estimación directa está dirigido a medir actitudes. Se partió de la identificación de la variable que se pretende medir, en este caso la VAP, así como de sus dimensiones e indicadores, definiendo, a su vez, el peso de cada categoría por el número de ítems, tomando en cuenta el peso en la relación con la variable VAP. Una vez realizada esta ponderación, se procedió con la redacción de los ítems que se utilizaron, generando un banco amplio que después se redujo en función de la pertinencia de cada uno de los reactivos.

Esta construcción consideró criterios básicos que todo ítem debe cumplir: sencillez, personalización, ausencia de ambigüedad, ausencia de carga emocional, no poner a la defensiva, neutralidad, evitar reacciones negativas y considerar una sola sentencia lógica (independencia) (Casas, Repullo y Donado, 2003). Es importante mencionar que la presentación de los ítems se realizó al azar y mezclando todas las dimensiones e indicadores, de manera que factores como el conservadurismo, la deseabilidad social, el sesgo de cortesía, la tendencia a repetir respuestas, el prestigio, la hostilidad hacia la temática y la influencia de las personalidades, quedaron controladas al máximo posible. Asimismo, y una vez redactados los ítems, se seleccionó el tipo de escala a utilizarse.

La escala de Likert es la escala que mayor validez y confiabilidad ha demostrado tener de entre todas las estudiadas (Likert, Guttman, Thurstone, Osgood), por la sencillez de su construcción y la consistencia demostrada con un número de ítems reducidos en comparación con las exigencias de las otras escalas. Esta escala establece el nivel de acuerdo o desacuerdo del respondiente en relación con cada una de las afirmaciones del cuestionario, valorando la conformidad del encuestado respecto a esas afirmaciones a través de índices que van desde el "Total acuerdo" hasta el "Total desacuerdo" (Casas, Repullo y Donato, 2002; Alaminos y Castejón, 2006; Maldonado, 2007). El presente estudio utilizará una escala de cuatro índices (Krosnick, Judd y Wittenbrink, en Albarracín, 2005): "Totalmente en desacuerdo", "Desacuerdo", "Acuerdo" y "Totalmente de acuerdo". Esta característica obliga a los encuestados a tomar posición respecto a cada afirmación del cuestionario, ya sea hacia lo favorable o a lo desfavorable.

Los pasos definidos para la construcción del cuestionario de estimación directa fueron:

1) Definir la actitud o variable a medir. 
2) Construir la estructura de la variable y sus componentes en base a la TPB.

3) Redactar ítems relacionados con la actitud desde la TPB. Se construyeron 100 ítems.

4) Definir la escala a utilizar y revisar la coherencia de cada ítem respecto a esta escala:

5) Revisar cada ítem respecto a su pertenencia a las dimensiones e indicadores.

6) Proceso de validación:

a. Tres validadores externos que realizaron observaciones respecto a: la correspondencia, entre el ítem y la categoría y subcategoría identificada, su construcción, su correspondencia con la base teórica, la adecuación a la escala propuesta y, finalmente, observaciones al cuestionario en general.

b. Se realizaron las correcciones correspondientes, resultando en 52 ítems.

c. Se aplicó una administración piloto en otra universidad.

d. Se revisaron los cuestionarios aplicados y se procedió a las últimas correcciones

Todos los ítems han sido verificados a través del método de componentes principales que permite transformar un conjunto de variables originales en un nuevo conjunto de variables denominadas “componentes principales” (Díaz, González, Henao y Pérez, 2012). Este método identifica las no correlaciones de los componentes de una muestra sin modificar el número original de variables del conjunto, facilitando la explicación de la varianza.

\section{Tabla 2}

Especificaciones para el cuestionario sobre actitudes hacia procesos de VAP

\begin{tabular}{|c|c|c|c|c|c|}
\hline SUBCATEGORÍA & COMPONENTE & $\begin{array}{l}\text { CANTIDAD } \\
\text { DE ÍTEMS }\end{array}$ & \multicolumn{2}{|c|}{ PONDERACIÓN } & No ÍTEM \\
\hline \multirow{5}{*}{$\begin{array}{c}\text { INTENCIÓN } \\
\text { COMPORTA- } \\
\text { MENTAL }\end{array}$} & Apertura & 5 & \multirow{5}{*}{18} & \multirow{5}{*}{$34.61 \%$} & $12 / 2 / 38 / 36 / 51$ \\
\hline & Derecho & 3 & & & $8 / 28 / 22$ \\
\hline & & & & & \\
\hline & Evaluación & 4 & & & $46 / \underline{49 * / 27 / 24}$ \\
\hline & Recursos & 6 & & & $39 / 19 / 50 / 48 / 23 / \underline{41}$ \\
\hline \multirow{4}{*}{$\begin{array}{c}\text { NORMA } \\
\text { SUBJETIVA }\end{array}$} & Autoridades & 3 & \multirow{4}{*}{17} & \multirow{4}{*}{$32.69 \%$} & $15 / 32 / \underline{4}$ \\
\hline & Docentes & 3 & & & $47 / 3 / \underline{29}$ \\
\hline & Estudiantes & 3 & & & $40 / 44 / \underline{33}$ \\
\hline & Institución & 3 & & & $25 / 1 / 30$ \\
\hline
\end{tabular}




\begin{tabular}{|c|c|c|c|c|c|}
\hline & Profesionales & 3 & & & $43 / \underline{21 / 10}$ \\
\hline & Sociedad & 2 & & & $42 / 35$ \\
\hline \multirow{4}{*}{$\begin{array}{l}\text { CREENCIA DE } \\
\text { CONTROL }\end{array}$} & Apertura & 5 & \multirow{4}{*}{17} & \multirow{4}{*}{$32.69 \%$} & $16 / 7 / 26 / 5 / 11$ \\
\hline & Evaluación & 2 & & & $\underline{6} / 31$ \\
\hline & Normativa & 2 & & & $20 / 52$ \\
\hline & Recursos & 8 & & & $\begin{array}{c}13 / 17 / 9 / 37 \\
/ 34 / 18 / 14 / 45\end{array}$ \\
\hline & & & & al de íter & 52 \\
\hline
\end{tabular}

\section{PRESENTACIÓN DE LOS RESULTADOS}

La aplicación del estudio de campo, la tabulación y análisis de los datos, proporciona los resultados que se presentan en el orden secuencial de la aplicación de los instrumentos: a) la encuesta de opinión; y b) el cuestionario de estimación directa.

\section{Resultados de la Encuesta de Opinión}

Lo que inicialmente se deseaba definir eran aquellas carreras que, por su proyecto académico construido desde el enfoque basado en competencias, podían estar potencialmente abiertas a procesos de VAP. Los resultados indican que la UCB tiene 73,3\% de sus carreras diseñadas desde un enfoque de formación basado en competencias, el EMI 90\% y la UMSA 51,9\%. A partir de estos resultados, se analizó el resto de la encuesta (tabla 3).

\section{Tabla 3}

Resultados en porcentajes obtenidos en la encuesta de opinión

\begin{tabular}{lllccc}
\hline & & $\mathrm{N}$ & $\begin{array}{c}\text { \% Respuestas } \\
\text { positivas }\end{array}$ & Desviación estándar & Error estándar \\
\hline Creencias respecto a la & UCB & 19 & $73,7 \%$ & $45,2 \%$ & $10,4 \%$ \\
inclusión en un plan de & EMI & 10 & $90,0 \%$ & $31,6 \%$ & $10,0 \%$ \\
licenciatura de personas & UMSA & 52 & $48,1 \%$ & $50,5 \%$ & $7,0 \%$ \\
con experiencia empírica & Total & 81 & $59,3 \%$ & $49,4 \%$ & $5,5 \%$ \\
\hline Planteamiento formal & UCB & 19 & $15,8 \%$ & $37,5 \%$ & $8,6 \%$ \\
sobre la posibilidad de & EMI & 10 & $10,0 \%$ & $31,6 \%$ & $10,0 \%$ \\
VAP & UMSA & 52 & $25,0 \%$ & $43,7 \%$ & $6,1 \%$ \\
\hline & VOLUMEN 24 No 3 SEPTIEMBRE-DICIEMBRE 2020 & 124 \\
\hline
\end{tabular}




\begin{tabular}{llcccc}
\hline & & $\mathrm{N}$ & $\begin{array}{c}\text { \% Respuestas } \\
\text { positivas }\end{array}$ & Desviación estándar & Error estándar \\
& Total & 81 & $21,0 \%$ & $41,0 \%$ & $4,6 \%$ \\
\hline Creencias sobre el aporte & UCB & 19 & $84,2 \%$ & $37,5 \%$ & $8,6 \%$ \\
al desarrollo productivo & EMI & 10 & $80,0 \%$ & $42,2 \%$ & $13,3 \%$ \\
de procesos de VAP & UMSA & 52 & $69,2 \%$ & $46,6 \%$ & $6,5 \%$ \\
& Total & 81 & $74,1 \%$ & $44,1 \%$ & $4,9 \%$ \\
\hline \multirow{2}{*}{$\begin{array}{l}\text { Viabilidad normativa para } \\
\text { la implementación de }\end{array}$} & UCB & 19 & $73,7 \%$ & $45,2 \%$ & $10,4 \%$ \\
VAP & UMSA & 52 & $51,9 \%$ & $48,3 \%$ & $15,3 \%$ \\
\cline { 2 - 6 } Viabilidad económica & Total & 81 & $59,3 \%$ & $50,5 \%$ & $7,0 \%$ \\
para la implementación de & EMI & 19 & $68,4 \%$ & $49,4 \%$ & $5,5 \%$ \\
VAP & UMSA & 52 & $80,0 \%$ & $47,8 \%$ & $11,0 \%$ \\
& Total & 81 & $46,5 \%$ & $42,2 \%$ & $13,3 \%$ \\
Viabilidad técnica para & UCB & 19 & $63,4 \%$ & $48,6 \%$ & $6,7 \%$ \\
procesos de VAP & EMI & 10 & $30,0 \%$ & $50,3 \%$ & $5,6 \%$ \\
& UMSA & 52 & $53,8 \%$ & $49,6 \%$ & $11,4 \%$ \\
& Total & 81 & $53,1 \%$ & $48,3 \%$ & $15,3 \%$ \\
\hline
\end{tabular}

Nota: Elaboración propia

En estos resultados destaca la EMI con una apertura importante hacia la inclusión de personas con experiencia empírica, a diferencia de la UMSA, que muestra una mayor cantidad de respuestas negativas. Ninguna de las tres universidades se planteó formalmente procesos de VAP, aunque la UMSA tiene algunas experiencias aisladas al respecto. Sin embargo, destaca la UCB con una mayor apertura en cuanto a la viabilidad normativa, la existencia de personal capacitado en estos procesos y las creencias de que estos procesos pueden resultar en un aporte al desarrollo productivo.

A partir de los resultados de esta encuesta y ponderando según la media, como se indicó en el acápite de método en la tabla 1, las carreras seleccionadas son:

- UCB: Administración de Empresas (310), Comunicación Social (310), Derecho (270), Diseño Gráfico (270), Ingeniería de Sistemas (270) y Psicopedagogía (250).

- UMSA: Estadística (310), Psicología (310), Electricidad (270), Lingüística e Idiomas (250) y Electrónica y Comunicaciones (250).

- EMI: Ingeniería Comercial Financiera (310). 
Se observan ciertas similitudes entre las universidades, particularmente entre la UCB y la UMSA, en cuanto a las carreras, que aún sin lograr ingresar en esta lista corta, mostraron apertura hacia los procesos de VAP. Tal es el caso de las carreras de Comunicación Social, Psicología, Ingeniería Industrial y Ciencias Políticas que tuvieron resultados positivos en ambas universidades, aunque no lograron la ponderación de cohorte (250 Ptos.).

Con estas carreras se procedió a la aplicación del cuestionario de estimación directa. Esta fase se inició con las tres universidades; sin embargo, la EMI no cumplió y no pudieron considerarse sus datos.

Al tratar la variable actitudes en el contexto de la TPB, se hace hincapié en que la medición de las dimensiones e indicadores debe permitir establecer si existe una actitud favorable de los académicos frente a la VAP. Para ello, se han definidos los componentes principales como índices dicotómicos de quienes están a favor y quiénes en contra. Esta conversión se ha efectuado en tres niveles: para la variable general y para las dimensiones e indicadores correspondientes.

El cuestionario de actitudes se aplicó a las carreras identificadas anteriormente. Los resultados generales, según dimensiones de análisis y universidad se presentan en la tabla 4, donde se compara la frecuencia por universidad y el porcentaje respecto a cada categoría y tomando en cuenta la relación dicotómica favorable-desfavorable.

Tabla 4

Distribución de académicos por dimensiones según universidad

\begin{tabular}{llcccccc} 
& & \multicolumn{2}{c}{ UCB } & \multicolumn{2}{c}{ UMSA } & \multicolumn{2}{c}{ Total } \\
\cline { 3 - 8 } & & Académicos & $\%$ & Académicos & $\%$ & Académicos & $\%$ \\
\hline Intención & En contra & 27 & $50,0 \%$ & 24 & $63,2 \%$ & 51 & $55,4 \%$ \\
Comporta- & A favor & 27 & $50,0 \%$ & 14 & $36,8 \%$ & 41 & $44,6 \%$ \\
mental & Total & 54 & $100,0 \%$ & 38 & $100,0 \%$ & 92 & $100,0 \%$ \\
\hline \multirow{2}{*}{ Norma } & En contra & 25 & $48,1 \%$ & 17 & $42,5 \%$ & 42 & $45,7 \%$ \\
Subjetiva & A favor & 27 & $51,9 \%$ & 23 & $57,5 \%$ & 50 & $54,3 \%$ \\
& Total & 52 & $100,0 \%$ & 40 & $100,0 \%$ & 92 & $100,0 \%$ \\
\hline \multirow{2}{*}{ Creencia de } & En contra & 32 & $60,4 \%$ & 11 & $32,4 \%$ & 43 & $49,4 \%$ \\
control & A favor & 21 & $39,6 \%$ & 23 & $67,6 \%$ & 44 & $50,6 \%$ \\
& Total & 53 & $100,0 \%$ & 34 & $100,0 \%$ & 87 & $100,0 \%$ \\
\hline
\end{tabular}

Nota: Elaboración propia 
Como puede observarse en la tabla 4, las tres dimensiones analizadas presentan comportamientos diferentes. Se observa que en la totalidad de académicos hay una tendencia a equilibrar sus posiciones en contra y a favor de los procesos de VAP. Esto puede deberse al desconocimiento sobre qué es la VAP y cómo se implementa. Los resultados cobran importancia en un análisis más minucioso por subcategorías, componentes y carreras.

En el caso de la Intención Comportamental, que analiza la predisposición a aceptar o no los procesos de VAP, a nivel descriptivo, la diferencia es mínima, pero con una inclinación hacia la no aceptación de los procesos de VAP en general, aunque más equilibrada en la UCB. La diferencia entre universidades muestra a la UCB con resultados totalmente equilibrados entre favorecer o rechazar estos procesos; en cambio en la UMSA, la diferencia es mucho más clara con una inclinación a estar en contra.

A nivel inferencial, la prueba obtenida $\left(\mathrm{Chi}^{2}=1,563 ; \mathrm{p}=0,211\right)$ demuestra que la opinión de cada universidad es independiente y, por lo tanto, no existe diferencia significativa respecto a la actitud hacia procesos de VAP en la dimensión Intención Comportamental.

Para la dimensión de Norma Subjetiva, relacionada a la percepción de los actores sobre cómo creen que personas relevantes y vinculadas a este ámbito podrían opinar, contrariamente a la anterior categoría, la tendencia a estar a favor de la inclusión de procesos de VAP, es más marcada, aunque tampoco de manera significativa. La Chi2 obtenida $\left(\mathrm{Chi}^{2}=0,283 ; \mathrm{p}=0,594\right)$ muestra un relacionamiento entre universidades con tendencias similares, ambas con esta postura a favor, pero de manera muy poco clara. En el análisis descriptivo por universidad, se observa que la UCB tiene resultados muy similares a la anterior categoría manteniendo un equilibrio entre estar en contra y estar a favor; sin embargo, a diferencia de lo que se observa en la categoría de Intención Comportamental, la UMSA, tiene una ligera inclinación a percibir que estas personas podrían estar a favor de los procesos de VAP.

Finalmente, en el caso de la dimensión de Creencia de Control, vinculada a la percepción de cuánto se considera que los proceso de VAP podrían estar bajo el control y la acción de los encuestados en sus universidades, se observa que inferencialmente sí existe una diferencia entre universidades, la Chi2 $\left(\mathrm{Chi}^{2}=6,508 ; \mathrm{p}=0,011\right)$ muestra que cada universidad tiene un comportamiento distinto. Los encuestados de la UCB perciben que el control de acciones relacionadas a los procesos de VAP, no están en sus posibilidades, a diferencia de los encuestados de la UMSA, cuya percepción de control se considera dentro de sus posibilidades. 
Por lo que la pertenencia a una determinada casa superior de estudios no es un factor determinante para pensar en una actitud positiva o negativa hacia la inclusión de procesos de VAP en las universidades. De igual manera, la Chi-cuadrado obtenida en cada uno de los componentes, relacionada con las universidades, muestra que esta tendencia se repite a un nivel más pequeño, sin grandes diferencias. (Probabilidad de rechazar la hipótesis que indica que las subcategorías están asociadas a la universidad, es mayor que 0,05)

\section{Tabla 5}

Resultados por Universidad y Carrera en las tres dimensiones

\begin{tabular}{|c|c|c|c|c|c|c|c|c|c|c|c|c|}
\hline & \multirow[b]{2}{*}{$\begin{array}{c}\text { Universidad/ } \\
\text { Carrera }\end{array}$} & \multicolumn{5}{|c|}{ UMSA } & \multicolumn{6}{|c|}{ UCB } \\
\hline & & 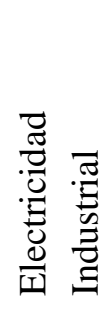 & 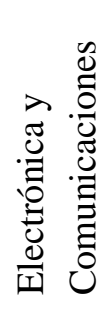 & 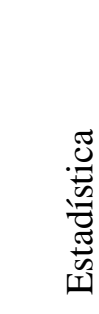 & 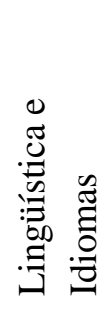 & $\begin{array}{l}\frac{\pi}{00} \\
\frac{0}{0} \\
\frac{0}{n} \\
0\end{array}$ & 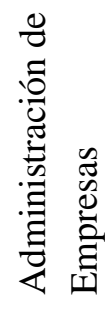 & 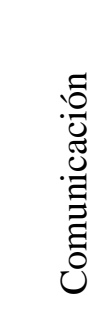 & $\begin{array}{l}\frac{0}{0} \\
\frac{d}{0} \\
\stackrel{0}{0}\end{array}$ & 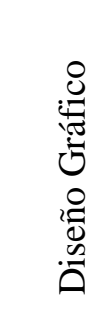 & $\begin{array}{l}\frac{\pi}{60} \\
0 \\
0 \\
\frac{0}{0} \\
0 \\
0 \\
0 \\
0 \\
0 \\
0\end{array}$ &  \\
\hline \multirow{4}{*}{ 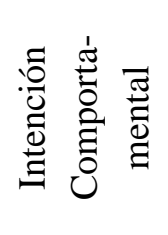 } & Apertura & 75 & 57,1 & 50 & 40 & 30 & 44,4 & 78,6 & 40 & 62,5 & 80 & 12,5 \\
\hline & Derecho & 25 & 57,1 & 83,3 & 40 & 33,3 & 33,3 & 64,3 & 50 & 50 & 60 & 50 \\
\hline & Evaluación & 12,5 & 71,4 & 83,3 & 60 & 22,2 & 66,7 & 64,3 & 50 & 50 & 100 & 37,5 \\
\hline & Recursos & 71,4 & 42,9 & 33,3 & 50 & 50 & 33,3 & 71,4 & 40 & 62,5 & 100 & 37,5 \\
\hline \multirow{6}{*}{ 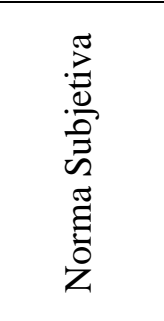 } & Autoridades & 85,7 & 71,4 & 50 & 50 & 60 & 22,2 & 46,2 & 10 & 37,5 & 40 & 25 \\
\hline & Docentes & 62,5 & 42,9 & 50 & 50 & 40 & 55,6 & 64,3 & 60 & 62,5 & 80 & 37,5 \\
\hline & Estudiantes & 75 & 85,7 & 66,7 & 50 & 60 & 33,3 & 78,6 & 60 & 50 & 60 & 37,5 \\
\hline & Institución & 28,6 & 42,9 & 66,7 & 20 & 30 & 55,6 & 76,9 & 60 & 50 & 100 & 75 \\
\hline & Profesionales & 37,5 & 28,6 & 33,3 & 40 & 30 & 55,6 & 78,6 & 40 & 62,5 & 60 & 37,5 \\
\hline & Sociedad & 62,5 & 85,7 & 66,7 & 50 & 70 & 22,2 & 71,4 & 50 & 37,5 & 100 & 75 \\
\hline \multirow{4}{*}{ 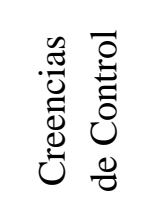 } & Apertura & 85,7 & 100 & 60 & 37,5 & 25 & 33,3 & 46,2 & 40 & 50 & 80 & 37,5 \\
\hline & Evaluación & 62,5 & 85,7 & 50 & 70 & 66,7 & 66,7 & 71,4 & 50 & 50 & 100 & 50 \\
\hline & Normativa & 75 & 85,7 & 66,7 & 80 & 80 & 77,8 & 64,3 & 60 & 75 & 100 & 50 \\
\hline & Recursos & 71,4 & 85,7 & 66,7 & 66,7 & 55,6 & 44,4 & 30,8 & 20 & 25 & 80 & 25 \\
\hline
\end{tabular}

Nota: Elaboración propia.

En la dimensión de Intención Comportamental, en cuanto a apertura, se observa que carreras, como electricidad industrial de la UMSA y Comunicación y Psicopedagogía de la UCB, tienen mejores perspectivas hacia procesos de VAP. En el caso del derecho de poblaciones no tradicionales a ingresar en la universidad, es la carrera de Estadística de la 
UMSA la que sobresale de entre todas. En el componente de evaluación, en términos de las creencias sobre la posibilidad de evaluar los aprendizajes previos, las carreras que se destacan son Electricidad y Comunicaciones y Estadística de la UMSA y Psicopedagogía de la UCB. Finalmente, respecto a la creencia sobre contar con los recursos técnicos y humanos necesarios para realizar procesos de VAP, destacan las carreras de Electricidad Industrial de la UMSA y Comunicación Social y Psicopedagogía de la UCB.

Las creencias respecto a la subcategoría de Norma Subjetiva, en tanto lo que consideran los encuestados que grupos relevantes podrían favorecer o estar en contra de procesos de VAP, se han considerado autoridades, docentes, estudiantes, las instituciones en sí mismas, profesionales en ejercicio y la sociedad en general. En cuanto a que autoridades universitarias podrían apoyar procesos de VAP, sólo las carreras de Electricidad Industrial y Electrónica y Comunicaciones de la UMSA responden positivamente. Para los docentes, sólo la carrera de Psicopedagogía de la UCB presenta creencias favorables. Sobre la opinión que podrían tener los estudiantes, sobresalen también las carreras de Electricidad Industrial y Electrónica y Comunicaciones de la UMSA y la carrera de Comunicación Social de la UCB. En cuanto a la institución y sus posibilidades de favorecer procesos de VAP, sólo carreras de la UCB responden positivamente: Comunicación Social, Psicopedagogía e Ingeniería de Sistemas. Respecto a cómo reaccionarían otros profesionales en ejercicio, sólo desataca positivamente la carrera de Comunicación Social de la UCB. Finalmente, para la percepción de la sociedad en general, destacan Electricidad y Comunicación y Psicología de la UMSA, y Comunicación Social, Psicopedagogía e Ingeniería de Sistemas de la UCB.

La última subcategoría se refiere a las consideraciones que pueda tener un sujeto frente procesos de VAP en función de la Percepción de Control que cree tener sobre los factores presentes o ausentes que puedan facilitar o impedir estos procesos. En este caso, se considerarán aspectos como la apertura hacia la implementación de VAP, la viabilidad de la evaluación, la normativa respectiva y los recursos necesarios.

Se puede observar que, en cuanto a la apertura hacia procesos de VAP, las carreras que destacan son Electricidad Industrial y Electrónica y Comunicaciones de la UMSA y Psicopedagogía de la UCB. Respecto a la posibilidad de tener control sobre la evaluación para procesos de VAP, están nuevamente Electrónica y Comunicaciones y Lingüística e Idiomas de la UMSA y Comunicación Social y Psicopedagogía de la UCB. Sobre la posibilidad de influir 
positivamente en la normativa que permita la VAP, todas las carreras, excepto Estadística, manifiestan creencias positivas en la UMSA y las carreras de Administración de Empresas, Diseño Gráfico y Psicopedagogía sobresalen en la UCB. Finalmente, en el caso del control de los recursos, nuevamente destacan Electricidad Industrial y Electrónica y Comunicaciones de la UMSA y Psicopedagogía de la UCB.

En general, las carreras que presentan tendencias favorables para la implementación de procesos de VAP son la carrera de Electricidad Industrial de la UMSA y Comunicación Social y Psicopedagogía de la UCB. Es con estas carreras que podría iniciarse procesos de capacitación y construcción de procedimientos para incluir a poblaciones adultas no tradicionales para su formación de grado.

En general (Figura 1), se observa una tendencia a presentar una actitud favorable a procesos de VAP, especialmente en las dimensiones Norma Subjetiva y Creencia de control. Esto podría representar que las creencias de los actores, pese a considerar positiva la opinión de otras personas significativas al respecto y de considerar que podrían presentarse las condiciones necesarias para que estos procesos se desarrollen, aún la tendencia a la acción misma es muy tibia, especialmente en la UMSA.

\section{Figura 1}

Comparación general de la tendencia a favor o en contra de cada categoría por universidad

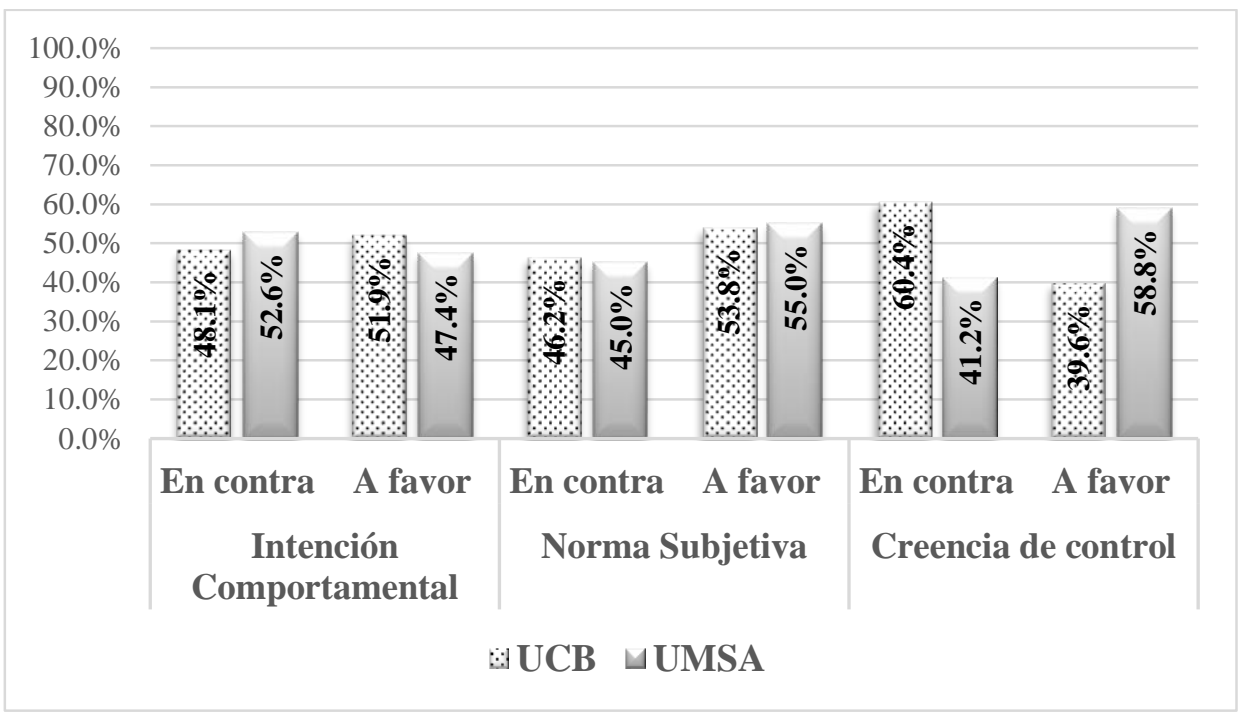

Nota: Elaboración propia 


\section{CONCLUSIONES Y RECOMENDACIONES}

Se ha logrado identificar y analizar las actitudes de los distintos actores vinculados a la educación superior, específicamente la de los académicos de las carreras seleccionadas, respecto a la inclusión en el SUB de personas con competencias empíricas a través de la VAP.

Los resultados alcanzados señalan que ni todas las carreras, ni todas las universidades del Sistema universitario han modernizado su oferta curricular desde un enfoque de formación basado en competencias, reduciendo las posibilidades de que puedan ingresar a procesos de VAP. La información obtenida con el primer instrumento evidencia que las carreras que se considera podrían incorporarse a procesos de VAP son pocas en ambas universidades y aún menores en la UMSA proporcionalmente en relación con el total (UCB: 5/20; UMSA: 5/50). Los resultados reflejan los modelos pedagógicos incorporados en cada una de las universidades. Mientras que la UCB se encuentra trabajando el enfoque de formación basado en competencias desde hace varios años, la UMSA recién está iniciando este proceso. Este aspecto no es menor, en tanto no existe la posibilidad de generar puentes entre aprendizajes empíricos y aprendizajes formales, sino no es a través de contar con unidades de análisis comunes, en este caso, las competencias.

Si bien, inicialmente, la UMSA se destaca porque ha tenido algunas experiencias previas y se ha planteado la inclusión de poblaciones no tradicionales a través de procesos de $\mathrm{VAP}$, es la que finalmente menor apertura denota. La universidad que se destaca en cuanto a la percepción de viabilidad tanto normativa, económica y técnica es la UCB, aunque cabe destacar que se trata de una universidad privada y autónoma cuya gestión de procesos como la VAP tendría que plantearse desde la autosostenibilidad. Esto es diferente a lo planteado por las experiencias de otras naciones, particularmente las europeas y mexicanas (Harris, Breier y Wihak, 2011; Duvekot, Coughlan y Aagaard, 2017, Unesco, 2019), que muestran alianzas con la industria para facilitar la realización de procesos de VAP, especialmente en lo referente su financiamiento.

El análisis realizado respecto a las actitudes, así como a sus características, evidencia, en general, un desconocimiento sobre la temática, como se permite deducir del análisis de relación obtenido entre las dimensiones y el factor Universidad. Sin embargo, se observan diferencias en las variables de análisis y sus dimensiones: Actitud comportamental, Norma 
Subjetiva y Creencia de control, debidas a las características de cada institución. En el caso de la UCB, existe mayor conocimiento sobre la temática y, por lo tanto, una posibilidad mayor de actuar en consecuencia, además de una percepción de autonomía respecto a las posibilidades de cada carrera; sin embargo, respecto a los recursos que se pueden controlar en términos de recursos humanos, técnicos y económicos, la UMSA tiene mayores posibilidades en tanto dependen del capital del Estado por ser una universidad pública que tiene la atribución de disponer de sus recursos con relativa autonomía, aspecto que la UCB no tiene al depender de la matrícula de sus estudiantes.

En general, las investigaciones muestran que los académicos presentan apertura hacia procesos de VAP cuando éstos no se incluyen en su propia universidad, particularmente debido al desconocimiento sobre estos procesos, así como a prejuicios respecto a lo que debe ser el proceso de aprendizaje para desarrollar competencias profesionales. Sin embargo, la apertura de instituciones de educación superior para estos procesos se ha incrementado de manera importante según los estudios revisados (Travers en Harris et. al., 2011).

La tendencia favorable hacia procesos de VAP de ciertas carreras respecto a otras, es muy clara y confirma investigaciones y experiencias anteriores que muestran que las carreras técnicas tienen mayor facilidad en la evaluación de las evidencias que demuestren el logro de los aprendizajes empíricos necesarios para continuar con su formación universitaria. Y a pesar de haber incluido muchos procedimientos que facilitan la VAP, pocas universidades han logrado desarrollar los mismos e incluirlos como procedimientos de acceso institucionalizado (Harris, 2011; Duvekot, Coughlan y Aagaard, 2017; Quevedo, 2019; Mercado, 2019).

En el análisis de ambas universidades y, considerando las diferencias entre ellas, es posible identificar que las creencias de control en la UMSA son más positivas respecto a otras dimensiones, aspecto que también podría estar asociado a la autonomía de esta universidad, especialmente en cuanto a que la sostenibilidad económica de los procesos de VAP no generan una gran preocupación, dado que sus recursos vienen del Estado.

Es relevante mencionar el efecto individual y social que tienen los procesos de VAP y que han sido investigados en otros países, desde algunos países de Latinoamérica, como Canadá, Estados Unidos, los miembros de la Comunidad Europea, países del Asia y Australia, (Harris, 2011; Duvekot, Coughlan y Aagaard, 2017). Hablamos de factores desconocidos para los facultativos bolivianos para quienes las discusiones respecto a procesos de VAP aún no se 
ha iniciado, por lo que el desconocimiento sobre este tema y sus impactos personales, institucionales y sociales aún son desconocidos. Sin embargo, el incremento que se ha dado en la mayoría de los países sobre estos procesos y las investigaciones que se han generado a partir de ello, estimulan la necesidad de continuar investigando y proponiendo la temática en agenda para que tanto instituciones como entidades de regulación gubernamental consideren a los procesos de VAP como mecanismos importantes para facilitar la inclusión y el desarrollo productivo de Bolivia.

Más aún desde el rol que juegan las universidades en el desarrollo productivo de sus países, dado que su compromiso social como organismo integrador con la sociedad, debe abrir las posibilidades de una institución que aborde problemas de impacto colectivo y frente a los cuales sea posible plantear soluciones (Rodríguez, 2015). Esto es lo que se pretende con la incorporación de procesos de VAP.

Los resultados alientan a iniciar procesos de sensibilización e información sobre la temática para proponer pasos concretos que permitan a las carreras que están más preparadas el comenzar a pensar seriamente en la incorporación de procesos de VAP que permitan la inclusión de poblaciones no tradicionales a la formación universitaria a nivel de licenciatura. Esta es la tarea de futuro que se precisa.

\section{REFERENCIAS}

Ajzen, I. (2011). The theory of planned behaviour: Reactions and reflections. Psychology \& Health, 26(9), 1113-1127. https://doi.org/10.1080/08870446.2011.613995

Ajzen, I., \& Fishbein, M. (2000). Attitudes and the Attitude-Behavior Relation: Reasoned and Automatic Processes. European Review Of Social Psychology, 11(1), 1-33. https://doi.org/10.1080/14792779943000116

Ajzen, I., Albarracín, D. (2007). Predicting and Changing Behavior: A Reasoned Action Approach. Prediction and Change of Health Behavior. Applying the Reasoned Action Approach 3-21.

Alaminos, A., \& Castejón Costa, J. (2006). Elaboración, análisis e interpretación de encuestas, cuestionarios y escalas de opinión. Universidad de Alicante.

Arias, F. (2012). El Proyecto de Investigación. Caracas: Epísteme 
Artigas, W. y Robles, M. (2010) Metodología de la investigación: Una discusión necesaria en Universidades Zulianas. Revista Digital Universitaria. 11(11). http://www.revista.unam.mx/vol.11/num11/art107/art107.pdf

Bharadwaj, Anandhi. (2000). Integrating positivist and interpretive approaches to information systems research: a Lakatosian model. Foundations of Information Systems. http://aisel.aisnet.org/cgi/viewcontent.cgi?article=1181\&context=amcis1996

Bizquera, R. (2009). Metodología de la Investigación Educativa. Madrid: La Muralla

Casanova, I. \& Canquiz, L. \& Paredes, I. \& Inciarte González, A. (2018). Visión general del enfoque por competencias en Latinoamérica. Revista de Ciencias Sociales24, 114125.https://dialnet.unirioja.es/servlet/articulo?codigo=7025273

Casas Anguita, J., Repullo Labrador, J., \& Donado Campos, J. (2003). La encuesta como técnica de investigación. Elaboración de cuestionarios y tratamiento estadístico de los datos (II). Atención Primaria, 31(9), 592-600. https://doi.org/10.1016/s0212$\underline{6567(03) 79222-1}$

Chilevalora.cl. (2019). Articulación con Formación ChileValora.https://www.chilevalora.cl/articulacion-con-formacion/formacion-tecnica/

Corral, Y., 2010. Diseño de cuestionarios para recolección de datos. Revista ciencias de la educación 152-168.http://hdl.handle.net/123456789/1985

Dávila, D. (2013). Aprendizaje a lo largo de la vida. Antecedentes y desafíos para la universidad de hoy. Revista Ciencia y Cultura, 17(30), 87-101

De la Fuente, S. (2011) Análisis de Componentes Principales. Universidad Autónoma de Madrid. Máster en Econometría.http://www.estadistica.net/

Demicheli, G., \& García, Á. I. (2016). Universidades Tradicionales y Calidad Institucional. Actitudes y Creencias de sus Estudiantes. Revista Iberoamericana de Psicología: $\begin{array}{lllll}\text { Ciencia } & y & \text { Tecnología, } & \text { 9(2), }\end{array}$ http://revistas.iberoamericana.edu.co/index.php/ripsicologia/article/view/671

Díaz Rodríguez, M., Ariza, A., Pérez, A., \& Mora, M. (2013). Introducción al análisis estadístico multivariado aplicado. Universidad del Norte.

Duvekot, R., Halba, B., Aagaard, K., Gabršcek, S. \& Murray, J. (2014). The Power of VPL. Validation of Prior Learning as a multi-targeted approach for access to learning 
opportunities for all. Inholland University AS \& European Centre Valuation Prior Learning. http://www.iriv.net/pdf/Biennale-book1-Power_of_VPL_final.pdf

European Centre for the Development of Vocational Training. (2009). European Guidelines for Validating Non-Formal and Informal Learning. Office. Luxembourg: Office for Official Publications of the European Communities. http://doi.org/10.2801/008370

Fernández, M. (2014).El aprendizaje informal en comunidades de práctica virtuales en la administración pública: evaluación y acreditación. Tesis doctoral. Universidad Autónoma de Barcelona. http://www.tdx.cat/handle/10803/286284

Fichten, C., Amsel, R., Asuncion, J. (2016). Theory of Planned Behavior: Sensitivity and Specificity in Predicting Graduation and Drop-Out among College and University Students?. Online Submission 15, 38-52. https://files.eric.ed.gov/fulltext/ED566977.pdf

Francis, A. J. J. (2004). Constructing Questionnaires Based on the Theory of Planned Behaviour A Manual for Health Services Researchers. https://doi.org/0-9540161-5-7

Hagger, M., Chan, D., Protogerou, C., \& Chatzisarantis, N. (2016). Using meta-analytic path analysis to test theoretical predictions in health behavior: An illustration based on metaanalyses of the theory of planned behavior. Preventive Medicine, 89, 154-161. https://doi.org/10.1016/j.ypmed.2016.05.020

Harris, J., Breier, M., \& Wihak, C. (2011). Researching the recognition of prior learning. Niace.

Hill, R., Fishbein, M., \& Ajzen, I. (1977). Belief, Attitude, Intention and Behavior: An Introduction to Theory and Research. Contemporary Sociology, 6(2), 244. https://doi.org/10.2307/2065853

Impulsaperu.gob.pe. (2019). Programa Impulsa Perú. http://www.impulsaperu.gob.pe/portal/el-programa

Krosnick, I.A., Judd. C.M. Y Wittenbrink, B. (2005). The Measurement of Attitudes. En Albarracin, D., Johnson, B., \& Zanna, M. (2005). The Handbook of Attitudes. (pp.2177). Mahwah, NJ: Lawrence Erlbaum Associates, Inc.

Liobikienė, G., Mandravickaitè, J., \& Bernatonienė, J. (2016). Theory of planned behavior approach to understand the green purchasing behavior in the EU: A cross-cultural study. Ecological Economics, 125, 38-46. https://doi.org/10.1016/j.ecolecon.2016.02.008 
López, N., \& Sandoval, I. (2006). Métodos y técnicas de investigación cuantitativa y cualitativa. Capítulo de libro. Universidad de Guadalajara. http://mail. udgvirtual. udg. mx/biblioteca/handle/20050101/1103

Maldonado, S. M. (2007). Manual práctico para el diseño de la Escala Likert. México: Trillas

Mercado, A. (2019). Entrevista para tesis doctoral [In person].

Ministerio de Educación (2017). Sistema Plurinacional de Certificación de Competenciashttp://www.minedu.gob.bo/sobre-spcc.html

Moscovici, S. (1991). Psicología social: influencia y cambio de actitudes: individuo y grupos. Barcelona: Paidós.

Organización de las Naciones Unidas para la Educación, la Ciencia y la Cultura. (2009). CONFITEA. VI Conferencia Internacional de Educación de Adultos: "Vivir y aprender paraun futuro viable: El poder del aprendizaje de adultos. http://www.Unesco.org/en/confinteavi

Organización de las Naciones Unidas para la Educación, la Ciencia y la Cultura. (2019). Global Inventory of Regional and National Qualifications Frameworks. Volume II: National and Regional Cases. UNESCO Institute for Lifelong Learning (LUI), UNESCO, European Training Foundation (ETF) and the European Centre for the Development of Vocational Training (Cedefop). 2, 74. https://doi.org/10.2816/841519

Quebedo, A. (2019). Entrevista para tesis doctoral [In person].

Rodríguez, A. (2015). 6. La Universidad y su compromiso social: una necesidad apremiante en Venezuela. Revista EDUCARE - UPEL-IPB - Segunda Nueva Etapa 2.0, 15(3), 128-

144. https://revistas.investigacion-upelipb.com/index.php/educare/article/view/208

Rodríguez, A. (2015). La Universidad y su compromiso social: una necesidad apremiante en Venezuela. Revista EDUCARE - UPEL-IPB - Segunda Nueva Etapa 2.0, 15(3), 128144. https://revistas.investigacion-upelipb.com/index.php/educare/article/view/208

Servicio Nacional de Aprendizaje (23 de septiembre de 2019). http://www.sena.edu.co/esco/Paginas/default.aspx

Singh, M. (2015). Global Perspectives on Recognizing Non-formal and Informal Learning Why Recognition Matters. UNESCO/UIL http://doi.org/10.1007/978-3-319-15278-3 
Steinmetz, H., Knappstein, M., Ajzen, I., Schmidt, P., \& Kabst, R. (2016). How Effective are Behavior Change Interventions Based on the Theory of Planned Behavior?. Zeitschrift Für Psychologie, 224(3), 216-233.https://doi.org/10.1027/2151-2604/a000255

Sulbarán, D. (2009). Medición de actitudes. Universidad Central de Venezuela. Facultad de Humanidades $\mathrm{y}$

Educación. https://psicoexperimental.files.wordpress.com/2011/03/dimas-sulbaran-escalas-deactitudes1.pdf

Tobón, S. (2008). Formación Basada en Competencias. Bogotá: EcoEdiciones.

Ubillos, S., Mayordomo, S. \& Pérez, D. (2003). Actitudes: definición y medición. Componentes de la actitud. Modelo de la acción razonada y acción planificada. En D. Páez, I. Fernández, S. CASAS \& E. Zubieta (Eds.), Psicología social, cultura y educación. Madrid: Pearson. 